\title{
Extratores aquosos para a caracterização química de substratos para plantas
}

\author{
Mônica Ferreira de Abreu; Cleide Aparecida de Abreu ${ }^{1}$; Isabele Sarzi²; Alceu Linares Padua Junior ${ }^{3}$ \\ ${ }^{1}$ Instituto Agronômico, Centro P\&D de Solos e Recursos Ambientais, C. Postal 28, 13012-970 Campinas-SP; ${ }^{2}$ Instituto Florestal, Seção \\ de Silvicultura; ${ }^{3}$ Aluno pós-graduação PG-IAC; monica@iac.sp.gov.br
}

\section{RESUMO}

Substratos são materiais que apresentam as mais variadas origens e composições, podendo ser orgânicos, minerais ou sintéticos. Avaliou-se métodos aquosos usando as proporções de 1:1,5; 1:2; $1: 2$ '; 1:5; 1:5' e 1:10 para a determinação de $\mathrm{pH}, \mathrm{CE}$, macro e micronutrientes em diferentes substratos comerciais e em componentes de substratos tendo-se como referência os resultados obtidos com o extrato de saturação. Foram utilizados nove substratos recomendados para as culturas de fumo, café, flores (2), florestais, citros e hortaliças (3) e onze componentes: casca de arroz carbonizada, turfa, turfa fibrosa, vermiculita, perlita, casca de pinus compostada (2), argila expandida, fibra de coco, cortiça e pó de xaxim. Foram determinados o $\mathrm{pH}$, condutividade elétrica (EC), macronutrientes e micronutrientes. Os resultados obtidos com $\mathrm{pH}$ e EC apresentaram os menores valores de coeficiente de variação $(\mathrm{CV})$ e para os micronutrientes foram muito próximos aos limites de detecção do aparelho. Os macronutrientes apresentaram coeficientes de variação entre 5,1\% e 47,2\%. O método 1:2 apresentou capacidade extrativa de macro e micronutrientes mais próxima daquela observada para o extrato de saturação e todas as soluções aquosas foram eficientes em avaliar o $\mathrm{pH}, \mathrm{CE}$ e macronutrientes dos diversos componentes e substratos.

Palavras-chave: substrato para plantas, análise química de substrato, macronutrientes, micronutrientes.

\section{ABSTRACT}

Water extracts for the chemical characterization of plant substrates

Substrates used to grow plants have different origins and compositions and can be organic, mineral or synthetic materials. In the present work the effect of different water extracts $(1: 1.5 ; 1: 2$; $1: 2^{\prime} ; 1: 5 ; 1: 5^{\prime}$; and $1: 10$ ) was evaluated and compared to saturated extracts for the determination of $\mathrm{pH}$, electric conductivity, macro and micronutrients in commercially available products and some of their components. The results were compared to those with saturated extracts. Nine substrates were evaluated, recommended for the cultivation of tobacco, coffee, flowers (2), forestry species, citrus and horticulture species (3), and eleven substrate components: carbonized rice hulls, peat, fiber peat, vermiculite, perlite, Pinus bark compost (2), expanded clay, coir (coconut fiber), cork and 'xaxim' or 'samambaia-açu' (Dicksonia sellowiana). Chemical analyses were made in the substrate extract solutions for the determination of $\mathrm{pH}$, electrical conductivity (EC), and macronutrient and micronutrient concentrations. The $\mathrm{pH}$ and $\mathrm{EC}$ data showed the lowest coefficients of variation. The micronutrient concentrations in the water extracts were very close to the equipment detection limits. The macronutrient concentrations showed coefficients of variation ranging from 5.1\% to $47.2 \%$. The method using the ratio $1: 2$ was better to obtain the saturation extracts for either macro or micronutrients. In spite of that, for all the other water extracts studied good results were obtained in the evaluation of $\mathrm{pH}, \mathrm{CE}$ and macronutrientes in the tested samples.

Keywords: substrates for plants, chemical analysis of substrates, macronutrients, micronutrients.

\section{(Recebido para publicação em 7 de janeiro de 2006; aceito em 18 de abril de 2007)}

$\mathrm{T}$ oda atividade na produção de mudas e plantas envasadas, principalmente de flores e plantas ornamentais, utiliza substratos. A demanda por substrato para o mercado da floricultura está em torno de 30 mil t ou $60 \mathrm{mil}$ $\mathrm{m}^{3}$ mensais (Bataglia \& Furlani, 2004).

O substrato pode ser formado de matéria-prima de origem mineral, orgânica ou sintética, de um só material ou mistura de diversos (Kanashiro, 1999). Os materiais orgânicos mais usados como substratos ou como componentes para substratos são turfa, casca de árvore picada, fibra de coco e, os materiais de origem mineral são vermiculita e perlita (Kampf, 2000).
A caracterização física e química dos substratos é necessária para a sua correta formulação e, também, para a recomendação e monitoramento das adubações nos sistemas de cultivo protegido. Contudo, até o presente, não há um método que seja totalmente aceito como o melhor para caracterizar quimicamente os substratos, embora haja consenso sobre quais análises necessitam ser feitas. Nos Estados Unidos há certa preferência pelo extrato de saturação, enquanto na Europa é mais comum o uso de extração com água em volumes fixos de 1:1,5, 1:5 e 1:10 (Abreu et al., 2002). Uma das grandes dificuldades em padronizar métodos está relacionada com a diversidade de materiais empregados como substratos para as plantas. $\mathrm{Na}$ Europa, com exceção da turfa, a maior parte dos materiais é praticamente inerte e, na maioria dos casos, os extratores aquosos são eficientes. No Brasil, os substratos utilizados em cultivos protegidos e semi protegidos são quase totalmente de origem orgânica, ou seja, materiais ativos. Esses materiais possuem elevada capacidade de troca de cátions (CTC), retendo os elementos para as plantas e, às vezes, as soluções aquosas não são tão eficientes. Santos (2005), avaliando a disponibilidade de $\mathrm{Cu}$ pelos métodos extrato de saturação e 1:1,5, observou que nenhum dos métodos 
apresentou correlação significativa entre $\mathrm{Cu}$ extraído da turfa, casca de pinus e fibra de coco com o $\mathrm{Cu}$ na parte aérea da rúcula.

Todos os métodos apresentam vantagens e limitações. Resultados obtidos com o extrato de saturação são menos reprodutíveis, por causa da dificuldade de se identificar o ponto final de diluição do substrato que define a saturação. A maior vantagem deste método esta no fato de determinar os nutrientes prontamente disponíveis às plantas (Bunt, 1986).

Furlani et al. (2005), utilizando os métodos aquosos 1:1,5;1:2;1:5;1:10 e a pasta de saturação incubados com fertilizantes minerais, concluíram que para o $\mathrm{pH}$ os valores do coeficiente de variação foram baixos $(\mathrm{CV}=6,8 \%)$.

De maneira geral, os resultados das soluções aquosas são afetados proporcionalmente em função das diluições. Furlani et al. (2005) obtiveram valores de 4,4; 3,3 e 1,4 mmol L-1 para a determinação de amônio na fibra de coco usando, respectivamente, os métodos $1: 1,5 ; 1: 2$ e 1:5. Resultados semelhantes foram obtidos para o $\mathrm{NO}_{3}^{-}, \mathrm{P}, \mathrm{K}, \mathrm{Ca}$, $\mathrm{Mg}$ e S. Outro aspecto importante é que quanto menor a diluição, mais próximos ficam os resultados daqueles obtidos com o extrato de saturação. $\mathrm{O}$ teor de Fe na fibra de coco extraído pela solução aquosa 1:1,5 foi de 4,2 $\mathrm{mg} \mathrm{L}^{-1}$, próximo àquele obtido pelo extrato de saturação de $5,7 \mathrm{mg} \mathrm{L}^{-1}$ (Abreu et al., 2004).

$\mathrm{Na}$ Holanda, tem-se adotado baixas diluições $(1: 1,5)$ e a padronização da umidade inicial, submetendo as amostras a uma pressão constante de $0,1 \mathrm{~kg}$ $\mathrm{cm}^{-2}$ antes de se proceder à diluiçãa das amostras (Sonneveld et al., 1974; Sonneveld, 1988). A indústria brasileira adota o extrato 1:2 para medir $\mathrm{pH}$ e CE (Abreu et al., 2002). A metodologia utilizando a proporção de 1:5 foi proposta pelo Comitê EUROPEÉN de NORMALISATION (CEN, 2003), onde a principal vantagem deste método é a possibilidade de se analisar vários tipos de substratos e componentes, tanto inertes como orgânicos.

No Brasil, ainda não há indicação do método mais adequado e existem poucas informações e muita controvérsia.
Falta uma definição do melhor procedimento e a sua padronização por meio de protocolos analíticos passíveis de uso nos laboratórios do país. Assim, o Instituto Agronômico de Campinas (IAC) foi escolhido pelo Ministério de Agricultura e Abastecimento para definir métodos eficientes em caracterizar quimicamente os substratos para as plantas e que sejam facilmente adaptados às condições de rotina dos laboratórios das empresas produtoras de substratos para plantas.

O objetivo deste trabalho foi avaliar métodos aquosos usando as proporções de $1: 1,5 ; 1: 2 ; 1: 2$ '; 1:5; $1: 5$ ' e 1:10 para a determinação de $\mathrm{pH}, \mathrm{CE}$, macro e micronutrientes em substratos comerciais produzidos e utilizados no Brasil e em componentes de substratos tendo-se como referência os resultados obtidos com o extrato de saturação.

\section{MATERIAL E MÉTODOS}

O experimento foi conduzido em condições de laboratório usando substratos comumente encontrados no comércio e componentes de substratos usualmente utilizados pelas empresas produtoras de substratos, que apresentavam diferentes características químicas e físicas. No comércio, foram selecionados nove substratos recomendados para as culturas de fumo, café, flores (2), florestais, citros e hortaliças (3) e onze componentes: casca de arroz carbonizada, turfa, turfa fibrosa, vermiculita, perlita, casca de pinus compostada (2), argila expandida, fibra de coco, cortiça e pó de xaxim. Nesses materiais foram avaliados os valores de $\mathrm{pH}, \mathrm{CE}$, macro e micronutrientes, em triplicata, empregando os seguintes procedimentos:

Extrato de saturação (ES) - Baseado em Warncke (1986). Em aproximadamente $400 \mathrm{~cm}^{3}$ de material (sem tratamento prévio) foi adicionada água deionizada até que a pasta apresentasse aspecto brilhante ou quando o sulco feito por uma espátula desaparecesse rapidamente. Após uma hora em repouso, foi conferido o ponto de saturação, adicionando-se mais água ou substrato conforme a necessidade. Após uma hora e meia de repouso, a pasta foi filtrada a vácuo em funil de Büchner, aplicando- se sucção até obter aproximadamente 25 $\mathrm{mL}$ de extrato.

Método 1:1,5 - Sugerido por Sonneveld \& Elderen (1994). Foi feito o tratamento prévio da amostra, onde cerca de $200 \mathrm{~cm}^{3}$ de material foram misturados com água deionizada até que, apertando-se levemente nas mãos a água escorresse por entre os dedos. Após esse ajuste a amostra foi colocada em dois anéis de $100 \mathrm{~cm}^{3}$ (correspondendo a 48 $\mathrm{mm}$ de diâmetro e $54 \mathrm{~mm}$ de altura), que receberam uma pressão de $10 \mathrm{kPa}$ (usando uma barra de ferro, correspondente ao peso de 1,8 kg). Os anéis foram separados e o conteúdo do anel inferior foi misturado com $150 \mathrm{~mL}$ de água deionizada e a suspensão agitada por 30 minutos usando agitador horizontal e posteriormente filtrada em papel de filtro, filtragem média (Nalgon 3550).

Método 1:2. Foram medidos 100 $\mathrm{mL}$ de substrato ou componente (sem tratamento prévio) e adicionados a 200 $\mathrm{mL}$ de água. A suspensão foi agitada por 20 minutos a $220 \mathrm{rpm}$ e filtrada em papel de filtro filtragem média (Nalgon 3550). Este método é rotineiramente usado pelas empresas de substrato do Brasil para determinação de pH e CE.

Método 1:2' - Recomendado por Sonneveld et al. (1990). O extrato foi obtido pela adição da amostra de substrato ou componente (sem tratamento prévio) em duas partes de água (200 $\mathrm{mL}$ ) até alcançar o volume de $300 \mathrm{~mL}$. A suspensão foi agitada por 20 minutos a $220 \mathrm{rpm}$ e filtrada em papel de filtro, filtragem média (Nalgon 3550).

Método 1:5 - O extrato foi obtido pela adição da amostra de substrato ou componente (sem tratamento prévio) em cinco partes de água $(250 \mathrm{~mL})$ até alcançar o volume de $300 \mathrm{~mL}$. A suspensão foi agitada por 20 minutos a $220 \mathrm{rpm}$ e filtrada em papel de fitro, filtragem média (Nalgon 3550).

Método 1:5' - Método oficial europeu (CEN, 2003). Neste método foram medidos $50 \mathrm{~mL}$ de amostra de substrato ou componente sem tratamento prévio e adicionados a $250 \mathrm{~mL}$ de água. A suspensão foi agitada por 20 minutos a 220 rpm e filtrada em papel de filtro, filtragem média (Nalgon 3550).

Método 1:10 - Procedimento empregado pela associação VDLUFA 
Tabela 1. Coeficientes de correlação entre $\mathrm{pH}, \mathrm{CE}$, macro e micronutrientes determinados pelo extrato de saturação (método de referência) e os demais métodos (Correlation coeficients among $\mathrm{pH}, \mathrm{CE}$, macro and micronutrients obtained through the saturation method and other ones). Campinas, IAC, 2005.

\begin{tabular}{lcccccc}
\hline \multicolumn{7}{c}{ Correlação entre o ES e os outros métodos } \\
\hline $\mathrm{CE}$ & $\mathbf{1 : 1 , 5}$ & $\mathbf{1 : 2}$ & $\mathbf{1 : 2}$ & $1: 5$ & $1: 5^{\prime}$ & $1: 10$ \\
$\mathrm{pH}$ & $0,944^{* *}$ & $0,830^{* *}$ & $0,933^{* *}$ & $0,894^{* *}$ & $0,910^{* *}$ & $0,755^{* *}$ \\
$\mathrm{NH} 4$ & $0,800^{* *}$ & $0,804^{* *}$ & $0,624^{* *}$ & $0,739^{* *}$ & $0,521^{*}$ & $0,759^{* *}$ \\
$\mathrm{NO} 3$ & $0,882^{* *}$ & $0,801^{* *}$ & $0,841^{* *}$ & $0,884^{* *}$ & $0,829^{* *}$ & $0,867^{* *}$ \\
$\mathrm{P}$ & $0,872^{* *}$ & $0,947^{* *}$ & $0,834^{* *}$ & $0,858^{* *}$ & $0,843^{* *}$ & $0,704^{* *}$ \\
$\mathrm{~K}$ & $0,834^{* *}$ & $0,844^{* *}$ & $0,809^{* *}$ & $0,834^{* *}$ & $0,786^{* *}$ & $0,826^{* *}$ \\
$\mathrm{Ca}$ & $0,969^{* *}$ & $0,929^{* *}$ & $0,947^{* *}$ & $0,926^{* *}$ & $0,935^{* *}$ & $0,930^{* *}$ \\
$\mathrm{Mg}$ & $0,955^{* *}$ & $0,943^{* *}$ & $0,913^{* *}$ & $0,935^{* *}$ & $0,891^{* *}$ & $0,590^{* *}$ \\
$\mathrm{~S}$ & $0,980^{* *}$ & $0,991^{* *}$ & $0,973^{* *}$ & $0,987^{* *}$ & $0,962^{* *}$ & $0,788^{* *}$ \\
$\mathrm{Na}$ & $0,966^{* *}$ & $0,941^{* *}$ & $0,928^{* *}$ & $0,947^{* *}$ & $0,918^{* *}$ & $0,701^{* *}$ \\
$\mathrm{~B}$ & $0,994^{* *}$ & $0,980^{* *}$ & $0,982^{* *}$ & $0,974^{* *}$ & $0,976^{* *}$ & $0,972^{* *}$ \\
$\mathrm{Cu}$ & $0,950^{* *}$ & $0,986^{* *}$ & $0,951^{* *}$ & $0,971^{* *}$ & $0,937^{* *}$ & $0,913^{* *}$ \\
$\mathrm{Fe}$ & $0,807^{* *}$ & $0,813^{* *}$ & $0,892^{* *}$ & $0,881^{* *}$ & $0,793^{* *}$ & $0,603^{* *}$ \\
$\mathrm{Mn}$ & $0,664^{* *}$ & $0,780^{* *}$ & $0,722^{* *}$ & $0,724^{* *}$ & $0,623^{* *}$ & $0,477^{*}$ \\
$\mathrm{Zn}$ & $0,776^{* *}$ & $0,880^{* *}$ & $0,906^{* *}$ & $0,925^{* *}$ & $0,772^{* *}$ & $0,867^{* *}$ \\
\hline
\end{tabular}

$*$ Significativo $5 \%$ de probabilidade; $* *$ Significativo $1 \%$ de probabilidade $(*$ Significant on the level of $5 \%$ probability; $* *$ Significant on the level of $1 \%$ probability).

(Normas de Federação dos Institutos para Pesquisas e Análises Agrícolas da Alemanha) e empregado pela Universidade Federal do RS (Fermino, 1996). As amostras de substratos ou componentes foram destorroadas manualmente. Depois, determinou-se a porcentagem de massa seca a $105^{\circ} \mathrm{C}$. As amostras foram secas ou umedecidas (conforme o caso), para ajustar a umidade a 50\%. Para obtenção do extrato, pesaram-se $20 \mathrm{~g}$ de material previamente tratado e adicionaram-se $200 \mathrm{~mL}$ de água. A suspensão foi agitada por 3 horas e depois em repouso por 24 horas e filtrada em papel de filtro, filtragem média (Nalgon 3550).

Com exceção do método da pasta de saturação, cujo $\mathrm{pH}$ foi determinado diretamente na pasta, em todos os demais procedimentos essa medida foi feita no filtrado, usando um eletrodo combinado de vidro. A condutividade elétrica foi medida à temperatura ambiente e o valor ajustado a $25^{\circ} \mathrm{C}$.

O nitrogênio foi destilado a partir da mistura do extrato com $\mathrm{MgO}$, para a determinação da forma amoniacal e depois com a liga de Devarda, para a forma de nitrato, utilizando a titulação potenciométrica com ácido sulfúrico (Bremner \& Keeney, 1966).
O potássio e sódio foram determinados por fotometria de chama e o fósforo, cálcio, magnésio, enxofre, boro, cobre, ferro, manganês e zinco por espectrometria de emissão óptica em plasma (ICP-OES).

$\mathrm{O} \mathrm{pH}, \mathrm{CE}$ e os teores de macro e micronutrientes dos vinte materiais extraídos pelos métodos $1: 1,5 ; 1: 2 ; 1: 2$; $1: 5 ; 1: 5$ ' e $1: 10$ foram correlacionados com aqueles extraídos pelo extrato de saturação, considerado padrão. A reprodutibilidade de cada método foi avaliada por meio do coeficiente de variação, considerando os 20 materiais, cujas análises químicas foram repetidas três vezes.

\section{RESULTADOS E DISCUSSÃO}

Os valores médios foram muito diferentes entre os métodos, indicando que estes têm capacidades de extração diferentes (Tabela 1). Dentre os métodos testados, o extrato de saturação e o 1:2' foram os que obtiveram maior capacidade de extração. De maneira geral, o extrato de saturação apresentou os maiores valores para os macronutrientes, enquanto que o 1:2' extraiu maiores quantidades de micronutrientes. Os teores muito próximos entre esses dois métodos devem-se ao fato da necessidade de se adicionar grande quantidade de material ao volume de água estabelecido de $200 \mathrm{~mL}$. Como os materiais apresentaram grande capacidade de absorção de água o ponto final ficou muito semelhante àquele obtido pelo extrato de saturação. Na Holanda, este método é empregado para análise de solo em cultivo protegido (Sonneveld et al., 1990), o qual apresenta características muito diferentes dos materiais utilizados neste estudo.

No que se refere ao fator de diluição entre os métodos, os resultados das soluções aquosas foram afetados proporcionalmente em função dessas diluições (Tabela 1), com exceção do método 1:10 cujo procedimento é muito diferenciado dos demais. Usando os métodos 1:1,5, 1:2 e 1:5, o teor de Mg nos extratos aquosos foram de 26,23 e $8 \mathrm{mg} \mathrm{L}^{-1} \mathrm{e}$ o de Fe de 2, 1,7 e 1,3 $\mathrm{mg} \mathrm{L}^{-1}$, respectivamente. Resultados proporcionais em função das diluições foram também obtidos por Furlani et al. (2005), que encontraram valores de 4,4, 3,3 e 1,4 mmol $\mathrm{L}^{-1}$ para a determinação de amônio na fibra de coco usando, respectivamente, os métodos 1:1,5, 1:2 e 1:5. Por outro lado, essa proporcionalidade do resultado em função da diluição contradiz o existente na literatura internacional. À medida que os extratos são mais diluídos, usando-se maiores volumes de água em relação a um volume fixo de substrato, os resultados não são afetados proporcionalmente (Johnson, 1980) provavelmente, porque os materiais aqui usados são bastante diferentes daqueles usados em outros países.

Os teores médios de micronutrientes dos diversos substratos e componentes, com exceção do B e do Fe, foram baixos (Tabela 1). Tal fato, deve-se aos baixos teores de $\mathrm{Cu}$, Mn e $\mathrm{Zn}$ nos materiais e/ou a baixa capacidade de extrativa dos métodos. A baixa capacidade de extração de $\mathrm{Cu}$ e $\mathrm{Zn}$ pode ser justificada pelo fato desses formarem complexos estáveis com ácidos fúlvicos e húmicos da matéria orgânica (Stevenson, 1991). Embora, o Fe e o Mn também possam formar complexos com a matéria orgânica, esses são menos estáveis que aqueles formados por $\mathrm{Cu}$ e $\mathrm{Zn}$ (Irving \& Williams, 1948). Portanto, métodos que empregam água como extrator não são capazes de extrair os micronutrientes 
ligados aos complexos estáveis com a matéria orgânica, apresentando, conseqüentemente, baixa capacidade extrativa.

Por meio do coeficiente de correlação entre os resultados do extrato de saturação e os demais métodos tem-se uma idéia do comportamento desses (Tabela 1). Em geral, os valores foram significativos a $95 \%$, sempre acima de 0,70 , indicando que os métodos estudados apresentaram comportamento semelhante ao extrato de saturação, definido como método de referência. Ainda nesta tabela, verifica-se que os micronutrientes, principalmente o Fe e o Mn, apresentaram os menores coeficientes de correlação com o extrato de saturação.

Entre os métodos, os menores valores de correlação foram observados para o método 1:10 (1). Provavelmente, devido ao fato do 1:10 apresentar algumas etapas diferentes dos demais métodos, como a padronização da umidade inicial da amostra de 50\%, o tempo de agitação da suspensão de três horas e o tempo de repouso de 24 horas.

O coeficiente de variação e o desvio padrão podem indicar a reprodutibilidade dos métodos. Essa característica é de grande importância para adoção de uma metodologia em análise de rotina. A variabilidade entre os resultados foi dependente do método e da característica analisada (Tabela 1). Dentre os procedimentos testados, a pasta de saturação e o método 1:10 foram os que apresentaram os mais baixos coeficientes de variação, com valores máximos de 38,5\% e 27,3\%, respectivamente, indicando melhor reprodutibilidade (Tabela 1). Por outro lado, o método 1:1,5 foi o de menor reprodutibilidade, com coeficiente de variação muito alto, atingindo $2 \%$ a $86 \%$. Os altos valores de coeficiente de variação encontrados para o método 1:1,5 podem ser atribuídos à avaliação empírica que se faz da umidade inicial da amostra.

Dentre as características analisadas, o $\mathrm{pH}$ foi o que apresentou o menor $\mathrm{CV}$, com uma amplitude de $1,0 \%$ a 2,5\%, seguindo-se da CE com CV de 5,2\% a $10,1 \%$. Esses valores podem ser considerados baixos devido à complexidade dos materiais analisados. Por outro lado, alguns elementos apresentaram altos coeficientes de variação e amplitude de variação, destacando-se os micronutrientes: B (8,9 a 31,9\%); $\mathrm{Cu}(6,2$ a $50,1 \%)$; Fe (19,2 a 86,3\%), Mn (11,8 a $46,7 \%)$ e $\mathrm{Zn}(12,2$ a $38,5 \%)$. As concentrações desses elementos nos diversos extratos estavam muito próximas ao limite de detecção do equipamento utilizado (ICP-OES), levando a erros analíticos e contribuindo para a imprecisão do método. Essa observação também foi feita por Bucher \& Schenk (2000) ao avaliarem o teor de $\mathrm{Cu}$ em turfa adubada usando os métodos: água, acetato de amônio, nitrato de amônio e cloreto de cálcio. A baixa concentração de micronutrientes nos extratos aquosos deve-se, provavelmente, ao baixo teor desses nos substratos e nos componentes.

O método 1:2 foi o que apresentou capacidade extrativa de macro e micronutrientes mais próxima daquela observada para o extrato de saturação. Todas as soluções aquosas foram eficientes em avaliar o $\mathrm{pH}, \mathrm{CE}$ e macronutrientes dos diversos componentes e substratos.

\section{AGRADECIMENTOS}

A FAPESP e PRODETAB pelo apoio financeiro.

\section{REFERÊNCIAS}

ABREU CA; FURLANI AC; ABREU MF; BATAGLIA OC; FURLANI PR. 2004. Micronutrient determination in different water extracts of coir fiber substrate incubated with mineral fertilizers. In: URRESTARAZU M. (ed.). INTERNATIONAL SYMPOSIUM ON SOILLESS CULTURE AND HYDROPONICS, 9. 2004, Almeria, Universidad de Almeria. p.113.

ABREU MF; ABREU CA; BATAGLIA OC. 2002. Uso da análise química na avaliação da qualidade de substratos e componente. In: FURLANI AMC; BATAGLIA OC; ABREU MF; ABREU CA; FURLANI PR; QUAGGIO JA; MINAMI K. (eds). ENCONTRO NACIONAL SOBRE SUBSTRATO PARA PLANTAS, 3, Campinas, 2002. Documentos IAC, 70, Campinas, Instituto Agronômico. p.17-28.

BATAGLIA OC; FURLANI PR. 2004. Nutrição mineral e adubação para cultivos em substratos com atividade química. In: BARBOSA JB; MARTINEZ HEP; PEDROSA MW; SEDIYAMA MAN. (eds). Nutrição e Adubação de Plantas cultivadas em substrato. Viçosa, Universidade Federal de Viçosa, p.106-128.
BREMNER JM; KEENEY DR. 1966. Determination and isotope-ratio analysis of different forms of nitrogen in soils: 3 . Exchangeable ammonium, nitrate, and nitrite by extraction-distillation methods. Soil Science Society of America Proceedings 30: 577-582.

BUNT AC. 1986. Problems in the analysis of organic and lightweight potting substrates. Hortscience 21: 229-231.

COMITÉ EUROPEÉN DE NORMALISATION - (CEN). 2003. EN 13652 - Soil improvers and growing media - Extraction of water soluble elements. Brussels, n.p.

FERMINO MH. 1996. Aproveitamento de resíduos industriais e agrícolas como alternativas de substratos hortícolas. Universidade Federal do Rio Grande do Sul, Porto Alegre. 110p. (Tese Mestrado).

FURLANI AC; ABREU MF; ABREU CA; FURLANI PR; BATAGLIA OC. 2005. Determination of available macronutrients, $\mathrm{Na}$, $\mathrm{Cl}, \mathrm{pH}$, and $\mathrm{EC}$ in a coir substrate incubated with mineral fertilizers. Acta Horticulturae 697: 109-115.

IRVING H; WILLIAMS RJP. 1948. Order of stability of metal complexes. Nature 162: 746747.

JOHNSON EW. 1980. Comparison of methods of analysis for loamless composts. Acta Horticulturae 99: 197-204.

KAMPF AN. 2000. SUBSTRATO. Produção comercial de plantas ornamentais. Agropecuária Guaíba, 254p.

KANASHIRO S. 1999. Efeitos de diferentes substratos na produção da espécie Aechmea fasciata (Lindley) Baker em vasos. 79p. ESALQ, Piracicaba, (Tese mestrado)

SANTOS PH. 2005. Avaliação de métodos de extração de micronutrientes em substratos para as plantas. Instituto Agronômico de Campinas, 67p. Campinas, (Tese mestrado)

SCHENK MK; BUCHER AS. 2000. Characterization of phytoavailable heavy metal, contents in compost-peat substrates, methods and toxicity levels. Acta Horticulturae 511: 173-183.

SONNEVELD C. 1988. Analytical methods for substrates in the Netherlands. Acta Horticulturae 221: 413-416.

SONNEVELD C; ENDE J; BES SS. 1990. Estimating the chemical compositions of soil solutions by obtaining saturation extracts or specific 1:2 by volume extracts. Plant and Soil 126: 169-175.

SONNEVELD C; ENDE J; DIJK PA. 1974. Analysis of growing media by means of a 1:1,5 volume extract. Communications in Soil Science and Plant Analysis 5: 183-202.

SONNEVELD C; ELDEREN CW. 1994. Chemical analysis of peaty growing media by means of water extraction. Communication on Soil Science and Plant Analysis 25: 3199-3208.

STEVENSON FJ. 1991. Organic-mattermicronutrients reactions in soil. In: MORTVEDT JJ; COX FR; SHUMAN LM; WELCH RM. (eds) MICRONUTRIENTS IN AGRICULTURE. Madison, Soil Science Society of America, p.145- 186.

WARNCKE DD. 1986. Analyzing greenhouse growth media by the saturation extraction method. HortScience 21: 223-225. 